\title{
Remarques générales sur les faciès magdaléniens du Bassin de l'Aude et du Roussillon
}

\author{
DOMINIQUE SACCHI *
}

\section{CADRE GÉOGRAPHIQUE}

La región concernée recoupe en grande partie la zone occidentale de l'ancienne province de Languedoc et la totalité du Roussillon. Elle est cantonnée au Nord par la Montagne noire, à l'Ouest par une ligne joignant le Seuil de Naurouze à la frontière espagnole, cette derniére formant la limite méridionale, à l'Est par le cours de l'Orb (fig. 1).

\section{HISTORIQUE DES RECHERCHES}

Dès le premier quart du XIXème siècle des naturalistes audacieux du Midi de la France s'appliquent à démontrer l'existence de l'homme fossile en cherchant ses restes dans les "cavernes à ossemens". Au cours de leurs fouilles, ces pionniers, au premier rang desquels Paul Tournal en 1827, vont rencontrer des niveaux archéologiques dont certains, nous le savons maintenant, sont représentatifs de la culture magdalénienne. Mais, exclusivement préoccupés par la quête d'ossements humains as-

* Chargé de recherche au CNRS, Laboratoire de Préhistoire, 21 Place de la Mutualité, 11.000 Carcassonne. 


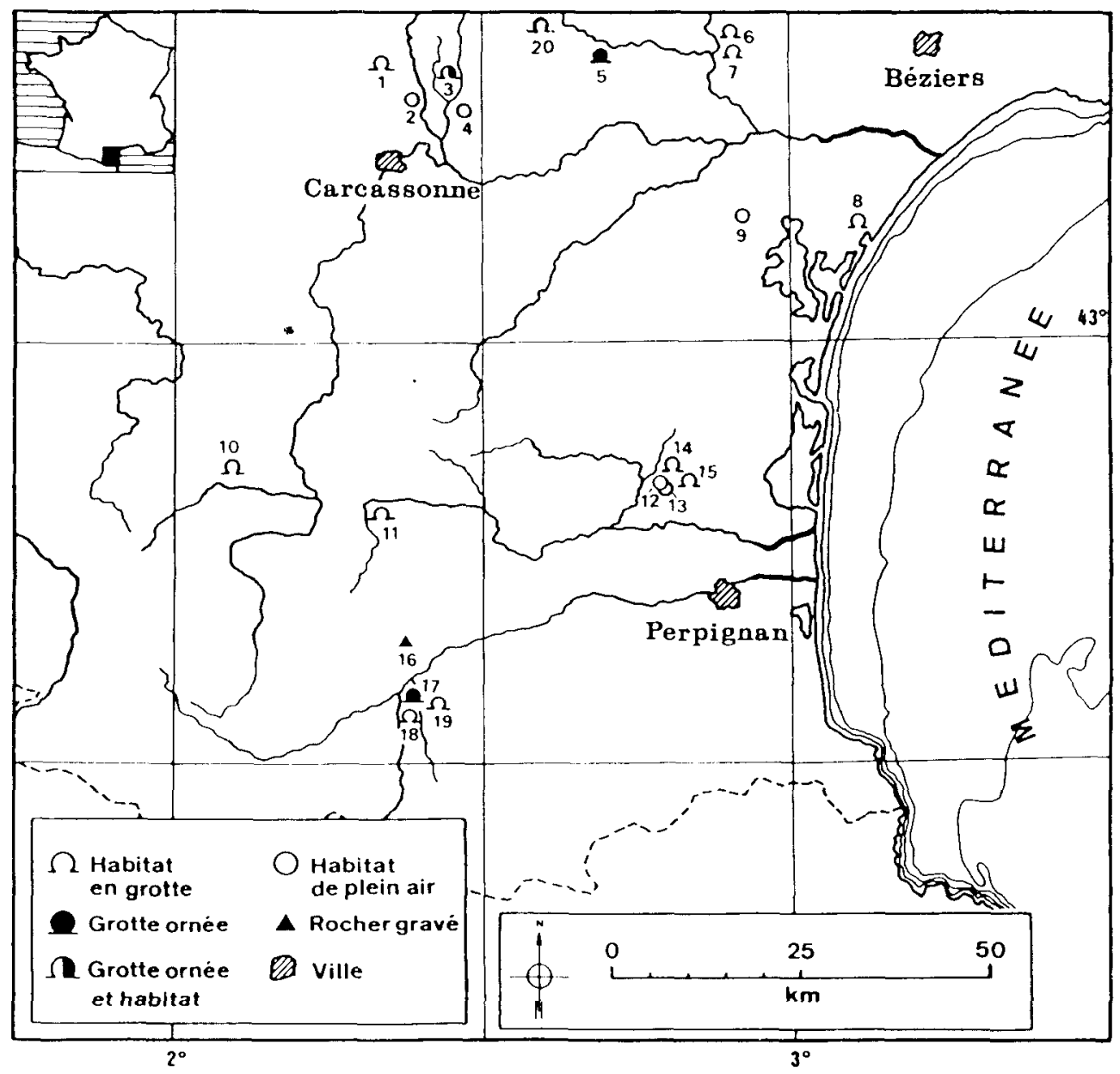

Fig. 1. Carte des sites archéologiques languedociens et catalans mentionnés dans le texte. $1=$ Canecaude, $2=$ Lassac, $3=$ Gazel, $4=$ La Rivière, $5=$ Aldène, $6=$ Petite grotte de Bize, $7=$ Tournal, $8=$ La Crouzade, $9=$ Fontlaurier, $10=$ Cauna de Belvis, $11=$ l'Oeil, $12=$ Rec de Penjat 806, $13=$ La Teulera, $14=$ Les Conques, $15=$ grotte du Harpon, $16=$ Fornols-Haut, $17=$ Cova Bastera, $18=$ Le Trou Souffleur, $19=$ Embulla, $20=$ Balma de l'Abeurador.

Solutréen final $=6,7,19 ;$ Magdalénien initial $=2,4,6,7$ (?); Magdalénien moyen $=1,3,6-8$; Magdalénien supérieur et final $=6-16,18,20$; faciès indéterminè $=5,17$. 
sociés à des espèces animales éteintes, ils méconnaîtront le caractère anthropogène de ces dépôts ainsi que les outillages qu'ils contiennent.

Ce n'est qu'à partir de 1867 , sous l'impulsion d'Edouard Filhol le père du Muséum d'Histoire Naturelle de Toulouse, et surtout d'Emile Cartailhac dont le rayonnement se fera longtemps sentir, que les recherches s'organisent et que sont identifiées les premières séries magdaléniennes. Au début du siècle et jusque vers les années trente, les études paléolithiques vont s'orienter, sous l'influence de Marcellin Boule, assurant ainsi le relais de son maître Cartailhac, vers une perspective stratigraphique avant que de marquer le pas. Dès lors les recherches s'accomplissent au hasard des découvertes. Citons notamment celles des gravures pariétales de Gazel en 1947 relevées par L. Méroc et G. Simonnet. L'année suivante est marquée par la parution de l'ouvrage de Marc Sauter "Préhistoire de la Méditerranée. Paléolithique - Mésolithique" dans lequel l'anthropologue genevois consacre quelques pages pertinentes et claires à la région qui nous intéresse, tirant même de l'anonymat ou de confidentielles publications locales quelques matériaux magdaléniens.

A partir de 1966, dans le cadre d'un programme de recherche concernant les cultures leptolithiques, il m'était donné d'entreprende l'étude du Magdalénien languedocien et nord-catalan. Recherche toujours en cours et dont un premier bilan fut publié en 1986.

\section{LE MAGDALÉNIEN ANCIEN OU BADEGOULIEN}

Peu après 17.000 B.P., selon la chronologie du radiocarbone, on constate la présence, en Languedoc, du Magdalénien ancien à raclettes. Alors qu'à Bize, dans la Petite grotte, les donnés stratigraphiques reproduisent le schéma classique du Badegoulien succédant directement au Solutréen final, la mesure d'âge du Solutréen final d'Embulla, arrêtée à $16.560 \pm 160$ B.P. (Sacchi, à paraître) montre, à moins de refuser cette estimation, la coexistence des deux faciès culturels (fig. 2). Le premier identifié tout d'abord dans le bassin de l'Aude (Sacchi, 1967, 1968, 1969), reconnu par la suite sur la rive droite du Bas-Rhône (Bazile, 1977) et tout récemment à Enlène (Clottes, à paraître), déborde donc largement des frontières méridionales qu'on lui assignait naguère (Sonneville-Bordes, 1966). 


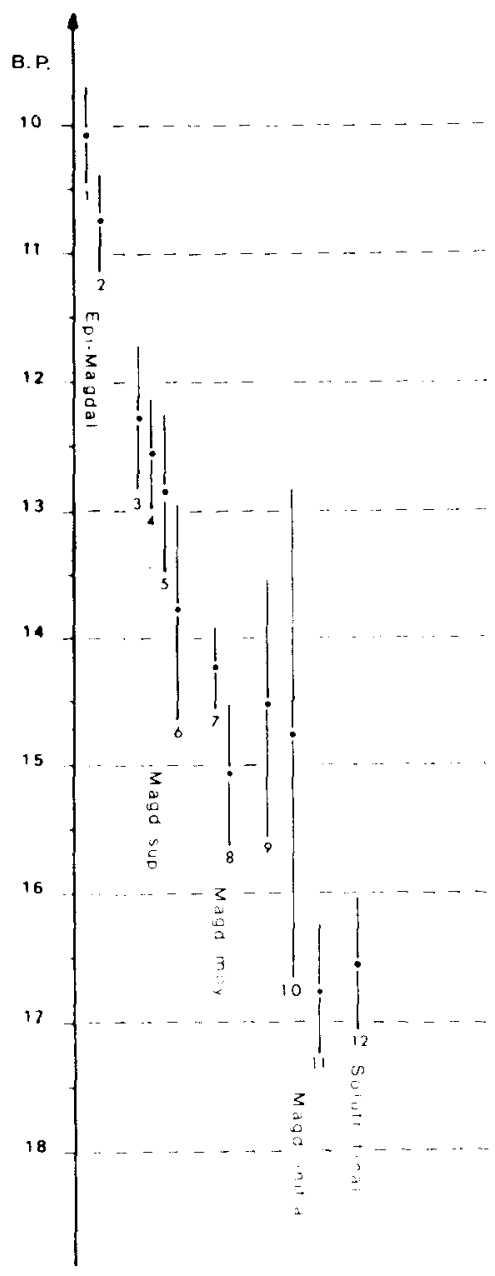

Fig. 2. Tableau des datations radiocarbones des faciès solutréen, magdaléniens et épimagdalénien individualisés dans la partie occidentale de la région de Languedoc-Roussillon. 1 = Gazel, couche 5: $10.080 \pm 190$ B.P. (Gif-2653); 2 = Gazel, couche $6: 10.760 \pm 190$ B.P. (Gif-2654); 3 = Cauna de Belvis, couche 3: $12.270 \pm 280$ B.P. (Gif-2950); 4 = Tournal, ensemble $I V$ - foyers noirs supérieurs : $12.550 \pm 210$ B.P. $(L y-1231) ; 5=$ Tournal, idem : $12.860 \pm 320$ B.P. (Ly-1894); $6=$ Tournal, idem : $13.790 \pm 400$ B.P. (Ly-1897); $7=$ Canecaude, couche II: 14.230 \pm 160 BP (Git-2708); $8=$ Gazel, couche $7: 15.070 \pm 270$ (Gif-2655); $9=$ Tournal, ensemble IV-niveau $G: 13.790 \pm 420$ (Ly-1232); $10=$ Tournal, idem : 14.770 \pm 970 BP (Ly-1675); $11=$ Lassac, Locus I - C2b : 16.750 \pm 250 B.P. (Git-2981); $12=$ Embulla, couche $1: 16.560 \pm 160$ B.P. (Git-6833). 
Deux des trois - peut-être quatre ${ }^{1}$ - gisements recensés correspondent à des stations de plein air. La plus vaste et la plus remarquable d'entre elles, Lassac, datée de $16.750 \pm 250$ B.P., occupait sur quelques dizaines d'hectares un large talweg et la base d'un glacis d'accumulation au pied d'une corniche calcaire dominant la rive droite de l'Orbiel, au Nord de Carcassonne.

Le décapage d'une structure pavée partiellement épargnée par le bulldozer demeure la seule fouille jamais réalisée sur ce site détruit avant que n'ait été menée à bien la vaste opération de sauvetage projetée.

Sous le pavage de galets prélevés par les Préhistoriques sur une nappe alluviale voisine gisaient des restes de rennes auxquels se mêlaient de nombreux vestiges d'industrie lithique et quelques rares pièces en matière osseuse.

Aux raclettes, pièces esquillées, lames appointées, burins transverses sur encoche ou troncatures latérales s'ajoutent d'abondantes lamelles à dos, dont quelques spécimens denticulés, des lamelles et triangles scalènes qui situent Lassac à un stade avancé de l'évolution du Magdalénien ancien, ce que confirme la datation radiocarbone. Ces particularités typologiques, encore accentuées par la domination constante des burins sur les grattoirs, sont communes au Magdalénien initial languedocien (fig. 3). II se distingue en cela de ses homologues du Périgord et d'lle-deFrance et annonce le Magdalénien classique.

La fraction osseuse de son équipement, relativement banale, comporte des armatures de sagaies biseautées unilatéralement (fig. $3, \mathrm{n}^{\circ}{ }^{0}$ ) ou appointées aux deux extrémites, des poinçons et des aiguilles à chas.

La station de Lassac, sans égale par son ampleur, doit certainement son existence aux migrations saisonnières du renne dont un gué voisin constituait sans doute le passage obligé. On en déplorera que davantage son anéantissement et donc la perte d'informations qui auraient permis d'entrevoir l'organisation d'un lieu évocateur de ces grands sites d'abattage et de dépeçage de rennes connus en d'autres temps, sous d'autres latitudes.

Les traces d'une activité culturelle et plus précisément artistique manquent totalement à Lassac. A Bize elles se manifestent bien modestement

Si l'on y ajoute la grotte Tournal dont le niveau G daté de $14.770 \pm 970$ et 14.530 $\pm 510 \mathrm{BP}$ (Tavoso, 1987) a livré une industrie ltihique réduite en nombre et restreinte en types mais qui par certaines de ces composantes et notamment les pièces esquillées, son mode de débitage, la nature et la patine du silex rapelle le Magdalénien ancien régional. 

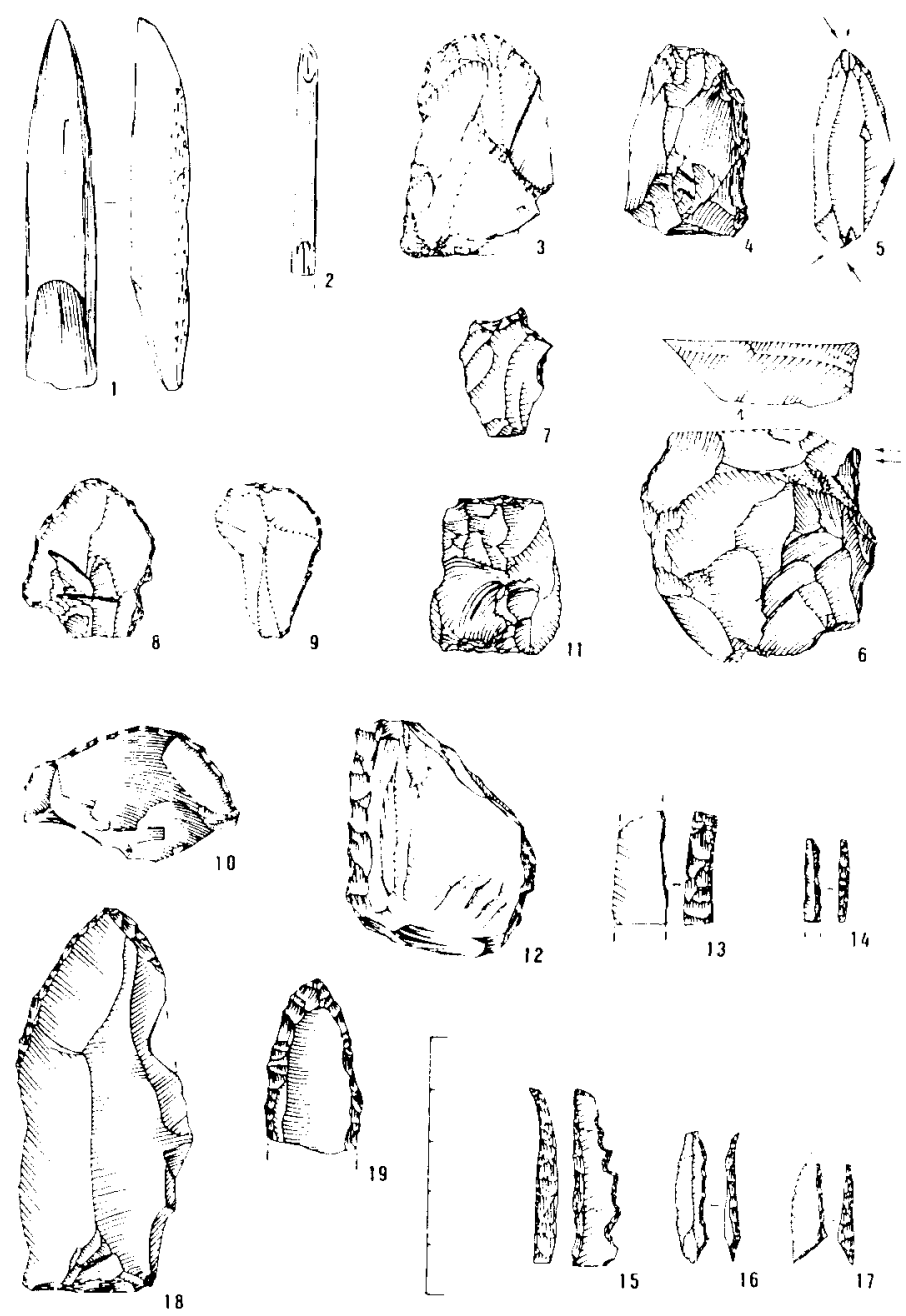

Fig. 3. Magdalénien ancien, industrie lithique et osseuse. 1 = armature de sagaie à biseau simple, 2 = fragments d'armature de sagaie gravée de signes, 3 = grattoir simple, 4 = grattoir caréné, $5=$ burin dièdre double, 6 = burin transversal sur encoche, $7=$ perçoir multiple, 8 à $10=$ raclettes, $11=$ pièce esquillée, 12 = racloir simple droit, 13 = lame à bord abattu dans le style gravettien, $14=$ microlamelle à dos, 15 = lamelle à dos denticulée, $16=$ lamelle scalène, $17=$ triangle scalène, 18 et $19=$ lames appointtées.

Petite grotte de Bize : 1 (couche 1 - fouilles Héléna), 2 et 10 (couche 5 - fouilles Genson); Lassac : 4, 6-8, 11, 15-17 (Locus 1 - fouilles Sacchi), 12, 14, 18, 19 (récoltes Durand); La Rivière : 3, 5, 9, 13 (récoltes $M$. Durand et sondage $D$. Sacchi). (Dessins de l'auteur). 
sous la forme de deux signes symétriques gravés sur une armature de sagaie (fig. $3, n{ }^{\circ} 2$ ), d'une élémentaire incisive de cervidé percée et de crayons de colorant rouge utilisés tout aussi bien à des tâches artisanales. Les habitants de Bize, stationnés sous le porche bien éclairé par la lumière diurne, visitèrent sans doute les profondeurs obscures de la cavité à la lueur de la lampe à graisse retrouvée. Moins spécialisés qu'à Lassac ces chasseurs ajoutèrent au Renne, toujours dominant, le Cerf, le Cheval, le Bouquetin, le Boeuf et le Lapin, d'après ce que nous savons des anciennes fouilles.

L'évolution floristique du domaine pyrénéen, connu par l'analyse pollinique des tourbières (Jalut et al. sous presse) n'autorise pas l'identification de l'épisode de réchauffement, connu ailleurs sous le nom d'interstade de Lascaux. II devient dès lors problématique de trouver une justification climatique à la prédominance des installations de plein air comme nous y avions un instant songé (Sacchi, 1986).

\section{LE MAGDALÉNIEN MOYEN}

Dès 15.000 B.P., la zone occidentale de notre région est occupée sans partage par les Magdaléniens. Ce qui ne semble pas le cas en Languedoc oriental. Là en effet et ce depuis le stade le plus ancien jusque vers 14.000 B.P. au moins, ils vont devoir partager le terrain et à coup sûr la vallée du Gardon avec les Salpétriens, sortes d'Episolutréens pour les uns ou d'Epigravettiens pour les autres. Toutefois, les habitats veritablement représentatifs du Magadalénien moyen s'agglomèrent tous dans le bassin moyen et inférieur de l'Aude, à la limite nord-est du domaine pyrénéen.

A l'instar de ce que l'on observe au coeur de cette dernière province, aucun des sites recensés ici n'échappe totalement au monde souterrain. Leurs hôtes, familiers de l'univers karstique, en investirent même les secteurs soustraits à la lumière du jour et s'accomodèrent parfois, singulièrement à Canecaude, d'espace où la station verticale s'avère impossible.

Par ailleurs, et cela peut surprendre pour des gisements apparemment inscrits sur les marges du foyer principal, ils se distinguent du lot par leurs datations radiométriques sensiblement plus élevées. Le plus ancien d'entre eux, la grotte Gazel, daté de $15.070 \pm 270$ B.P., devance de huit siècles et demi, selon la moyenne des estimations, l'établissement 

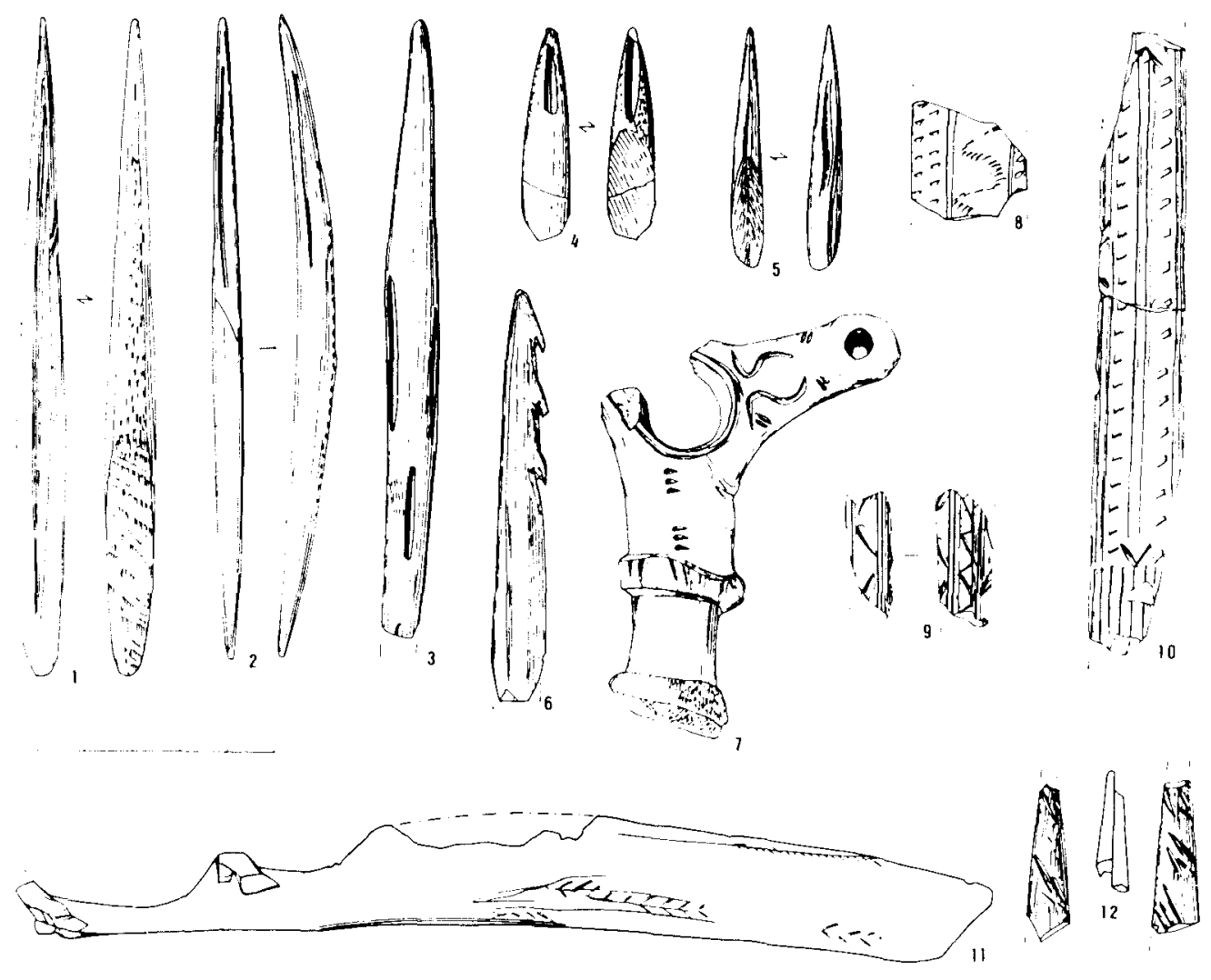

Fig. 4. Magdalénien moyen, industrie osseuse. 1 = armature de sagaie à biseau simple, 2 = armature de sagaie bi-pointe, 3 = armature de sagaie biconique, 4 5 = armatures de sagaies du type "Lussac-Angles", 6 = armature barbelée ou protoharpon, 7 = bâton à trou orné et muni d'une perforation de suspension, $8=$ fragment de lissoir décoré, $9=$ tronçon de baguette profondément gravée, $10=$ baguette demi-ronde ornée, $11=$ corps de propulseur primitivement orné d'une figure sculptée d'ongulé dont ne subsiste que les quatre pieds, 12 = éléments de baguettes demi-rondes gravées, accolables par la face plane.

Canecaude : $2-5$ (couche II, fouilles D. Sacchi); Gazel : 1, 6, 9, 11, 12 (couche 7, fouilles D. Sacchi), 7, 10 (fouilles S. Nouvian). (Dessins de l'auteur). 
de Canecaude. Le premier répond sûrement à la notion de super-site ou lieu de rassemblements périodiques développée par les préhistoriens de langue anglaise. Ses $1.000 \mathrm{~m}^{2}$ de surface d'habitat (fig. 6), son sanctuaire aux parois gravées, l'abondance et la variété des équipements en roche siliceuse, en matière dure animale et la profusion des vestiges faunistiques abandonnés par les occupants, l'origine atlantique de certains produits manufacturés en soulignent en tous les cas l'importance.

La seconde station d'une capacité beaucoup plus réduite, implantée dans une vallée étroite et encaissée, servait de gîte aux individus chassant le renne dans les parages. D'après l'évaluation de l'âge des nombreux spécimens de cet ongulé mis au jour à la fouille, $\mathrm{M}$. Maurel (in Sacchi, 1979) conclut à une fréquentation de la station échelonnée sur l'année. En revanche, B. Gordon (1988), dont l'étude n'a porté que sur un seul sujet âgé de 24 mois, fixe l'occupation à la mi-été et admet la proximité du lieu de mise bas.

L'industrie lithique se différencie de celle du stade précédent par son mode de débitage, les faibles dimensions de ses produits bruts ou façonnés tout autant que par l'abandon ou plus rarement l'adoption de certains types d'outils. Une production systématique de lames et lamelles traduit l'utilisation rationnelle d'une matière première rare car généralement étrangère. Au silex thanétien local, ordinairement taillé par les Badegouliens et maintenant réduit au rang de matériau d'appoint, se substituent des petits galets et rognons de silex blond, brun et gris qu'une exploitation économe a bien souvent réduit à l'état de minuscules nucléus diminutifs.

Mais si les techniques de débitage mises en oeuvre par les Magdaléniens moyens diffèrent de celles qu'utilisaient leurs prédécesseurs, la pratique en sera maintenue par leurs successeurs qui perpétueront aussi l'usage des mêmes instruments. L'équipement lithique demeure donc impropre à caractériser de façon convaincante cette phase de la civilisation magdalénienne. En revanche, nous trouvons ces éléments distinctifs parmi les fruits du travail de l'os et du bois de renne, alors à son apogée. Ce sont les propulseurs sculptés de figures animales, les contours découpés en forme de têtes de chevaux, les baguettes demi-rondes décorées de tubercules alignés, les spatules gravées, les proto-harpons, les longues sagaies à biseau simple, soit, pour la plupart, les composants essentiels de la panoplie du Magdalénien IV, selon la définition de Breuil.

On constate en outre la présence de la pointe de sagaie dite de Lussac-Angles, considérée comme un atribut déterminant du Magdalénien III dans le dispositif chronologique traditionnel. Dans la région con- 

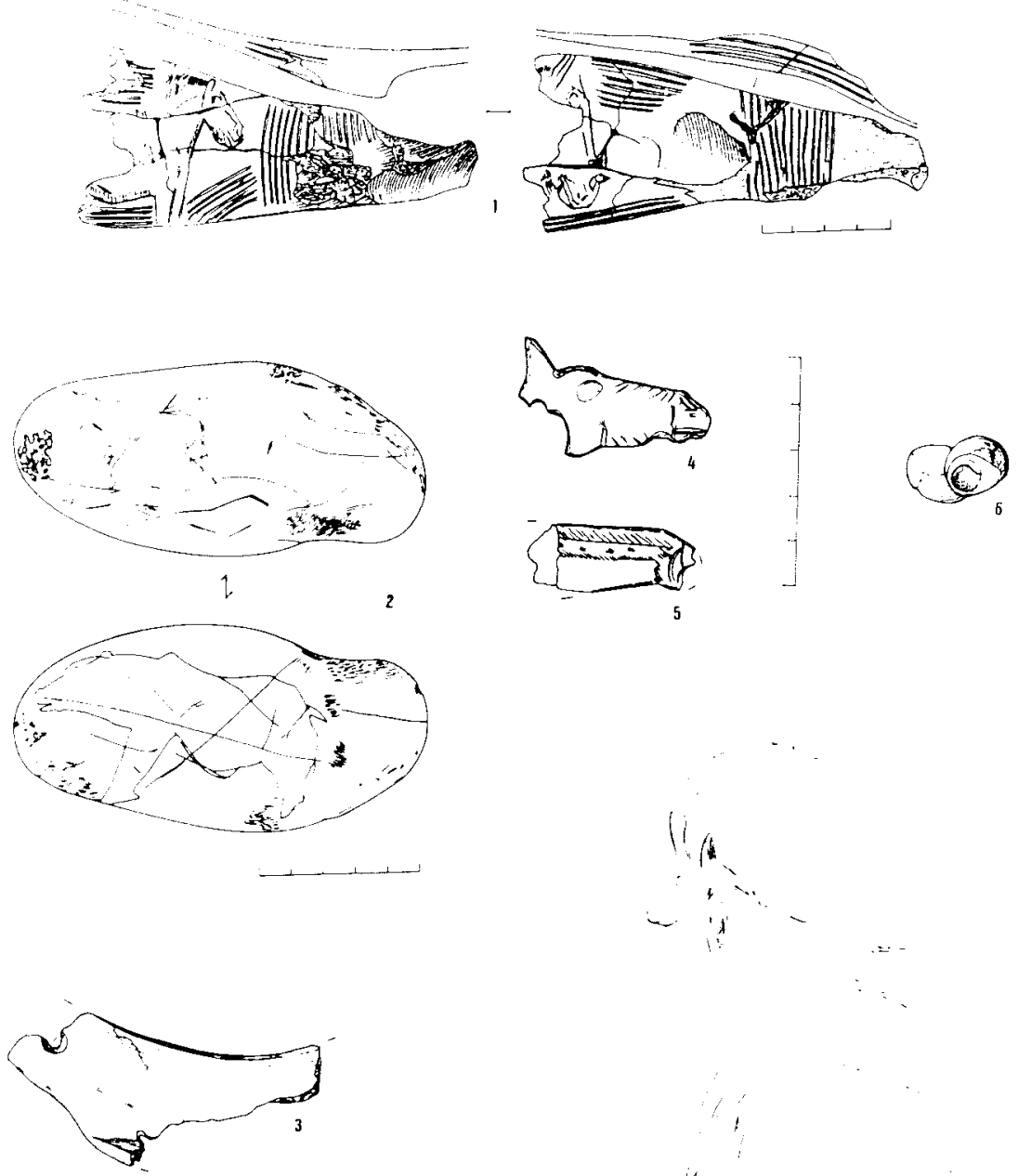

Fig. 5. Magdalénien moyen, art animalier et parure. 1 = chevaux enfermés dans des réseaux de traits gravés sur fragment de mandibule de cheval, 2 = silhouettes d'aurochs et d'ours gravées sur galet-retouchoir, 3 = ébauche de contour découpé dans un stylohyal d'os hyoïde de cheval, 4 et $5=$ contours découpés en forme de têtes de chevaux, $6=$ littorines obtuses artificiellement percées retrouvées soudées dans leur position d'enfilage, $7=$ bouquetin gravé sur paroi. Grotte de la Crouzade : 5 (fouilles Genson), grotte Gazel: 1 (fouilles Nouvian), 2-4 et 6 (couche 7, fouilles Sacchi), 7 (relevé Sacchi). (Dessins de l'auteur). 
sidérée, ce type d'armature, dominant à Canecaude, ne se dissocie jamais du matériel typique du Magdalénien classique régional. On ne peut donc lui accorder une valeur chrono-culturelle précise au sein de cette séquence. Ce n'est pas le cas de certaines ornementations, par exemple des festons d'incisions fréquemment encadrés de lignes d'entailles (fig. 4, n. ${ }^{\circ} 8$ ). Ce motif privilégié des lissoirs et spatules, qui retint l'attention à diverses reprises (Graziosi, 1960; Bahn, 1982; Sacchi, à paraître b) figure en bonne place dans la thématique pyrénéenne.

A l'exception des tête d'équidés déjá mentionnées les objets à suspendre, qu'il s'agisse des dents animales, des coquilles marines et des perles en jayet, sont communs à tous les facies. Notons toutefois que les tests de Littorina obtusata et Littorina littorea, cette dernière espèce ne pouvant provenir que du rivage atlantique, se rencontrent de préférence dans les dépôts du Magdalénien moyen (fig. $5, \mathrm{n}^{\circ}{ }^{\circ}$ ).

L'art mobilier, indissociable dans bien des cas de l'outillage ou de l'armement, fait également la place à des variétés d'objets dont la fonction ou l'usage demeure inconnu. Si ce n'est pas le cas des galeis gravés de silhouettes animales utilisés comme compresseurs et retouchoirs (fig. 5, n. $^{\circ} 2$ ), on s'interroge sur le rôle des os plats où se développent parfois de remarquables dessins animaliers (fig. 5, n. ${ }^{\circ} 1$ ). Quant aux plaquettes de grès et autres roches fossiles, si abondantes en Couserans et en d'autres points du territoire magdalénien, elles demeurent absentes des sites en question.

Au nombre des grottes ornées seule Gazel, gravée de bouquetins et d'équidés, peut être attribuée sans risque d'erreur majeure au Magdalénien moyen. II en va autrement de la grotte d'Aldène aux images quelque peu déconcertantes ou de la Cova Bastera avec ses chiches poncuations et bâtonnets peints en rouge. Quant au signe rouge, peu lisible et inédit, de Canecaude, il est bien évidemment tentant de la relier à la couche II magdalénienne qui seule livra des colorants de cette teinte.

Omniprésent à Canecaude et dominant à Gazel, le Renne s'affirme comme le gibier d'élection des Magdaléniens moyens. Mais dans le second gisement il laisse une large place au Cerf, au Cheval ainsi qu'aux léporidés particuliérement abondants.

Les données paléobotaniques (Renault-Miskovsky in Sacchi, 1979 : Vernet idem), paléozoologiques (Maurel, op. cit.; Vilette, 1983; DupuisPerez, 1986), sédimentologiques (Brochier, 1978) s'accordent assez bien, tant à Canecaude qu'à Gazel, à mettre en évidence un paysage largement ouvert, mais non dépourvu de zones boisées protégées, sous l'emprise de conditions climatiques rigoureuses. 

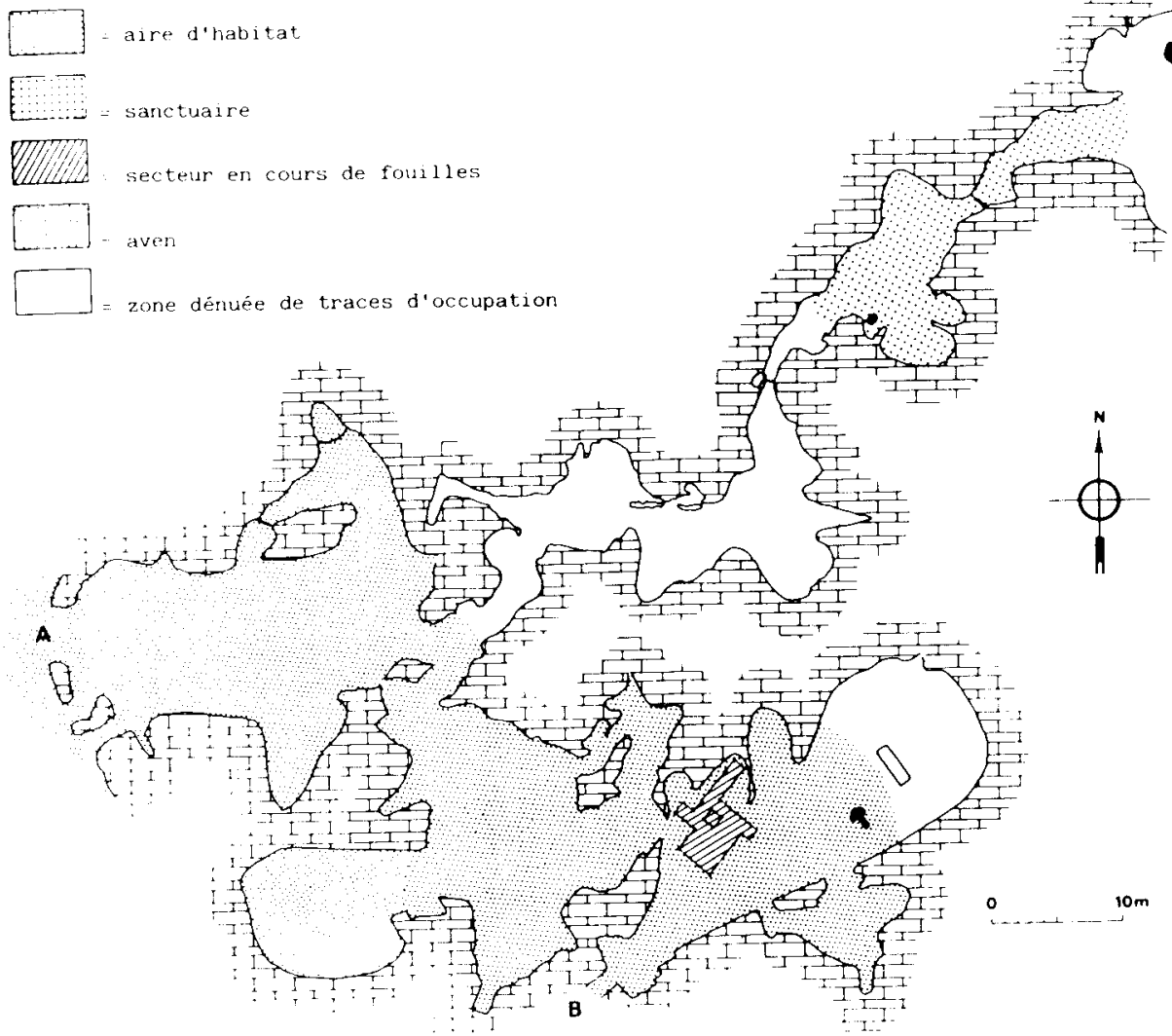

Fig. 6. Magdalénien moyen, un site de rassemblent : la grotte Gazel. Selon les estimations minimales, la surface d'occupation atteignait $1000 \mathrm{~m}^{2}$. A et B $=$ entrées. 


\section{LE MAGDALÉNIEN SUPÉRIEUR ET FINAL}

En son stade supérieur et ultime la civilisation magdaléniense intensifie son emprise territoriale comme en témoigne la douzaine de sites recensés à ce jour. Ces habitats connaissent une distribution large et variée au point d'investir même les reliefs montagneux de moyenne altitude (Belvis, $960 \mathrm{~m}$ ). Si neuf d'entre eux occupent des cavités naturelles, les installations en milieu obscur sont désormais exceptionelles.

La multiplication des établissements, précédement ignorés, dans la majorité des cas, des Magdaléniens moyens, correspond ici comme ailleurs à une vraisemblable croissance démographique. Cet accroissement de la population aurait entraîné du même coup un fractionnement des communautés pour une meilleure exploitation des ressources naturelles. C'est dumoins ce qu'il semble possible de déduire du faible facteur d'habitabilité de la plupart des stations. Les plus grandes d'entre elles, qu'il est impossible d'évaluer précisément du fait des fouilles anciennes, s'inscrivent dans les limites de certaines cavernes occupées durant la phase antérieure (Tournal, La Crouzade) ou se développent à l'air libre aux abords de quelque source ou ruisseau (Fontlaurier).

Sur le plan de la chronologie, le Magdalénien supérieur s'enchaîne bien avec le Magdalénien moyen dont il constitue le prolongement logique à partir de 13.800 B.P. jusque vers 12.300 B.P. selon les datations radiocarbones extrêmes (fig. 2).

$\mathrm{Si}$, comme nous l'avons déjà dit, ces deux stades ne se différencient guère au niveau de la fraction lithique de l'équipement, on note toutefois une plus large utilisation des roches siliceuses locales. En revanche le Magdalénien final, se distingue par le développement des petites pointes à dos (fig. 7, n. ${ }^{\circ} 3-6$ ), l'accentuation, particulièrement aiguë à Belvis, des processus de microlithisation et de géométrisation (fig. $7, n .{ }^{\circ} 7-10$ ) et la présence occasionalle de rares pointes à cran (fig. $7, n .^{\circ} 1$ et 2 ).

Là encore c'est à travers les produits de l'industrie osseuse ses acquisitions et ses abandons que l'on mesure le mieux les marques du changement. Alors que dans un premier temps subsistent, à Bize et à la Crouzade, les baguettes demi-rondes, surgissent simultánément les tetes de harpons à simple et double rangées de barbelures (fig. $7, \mathrm{n} .^{\circ} 11,16$ 19). Les armatures de sagaies à biseau double (fig. $7, n .{ }^{\circ} 20$ ) supplantent les spécimens biconiques et à simple biseau qui disparaîtront ensuite. Au terme de cette période des petites pointes à base taillé et rompue (fig. 7, n. ${ }^{\circ}$ 13-15), dont nous avons reconstitué le mode de production (Sacchi, 

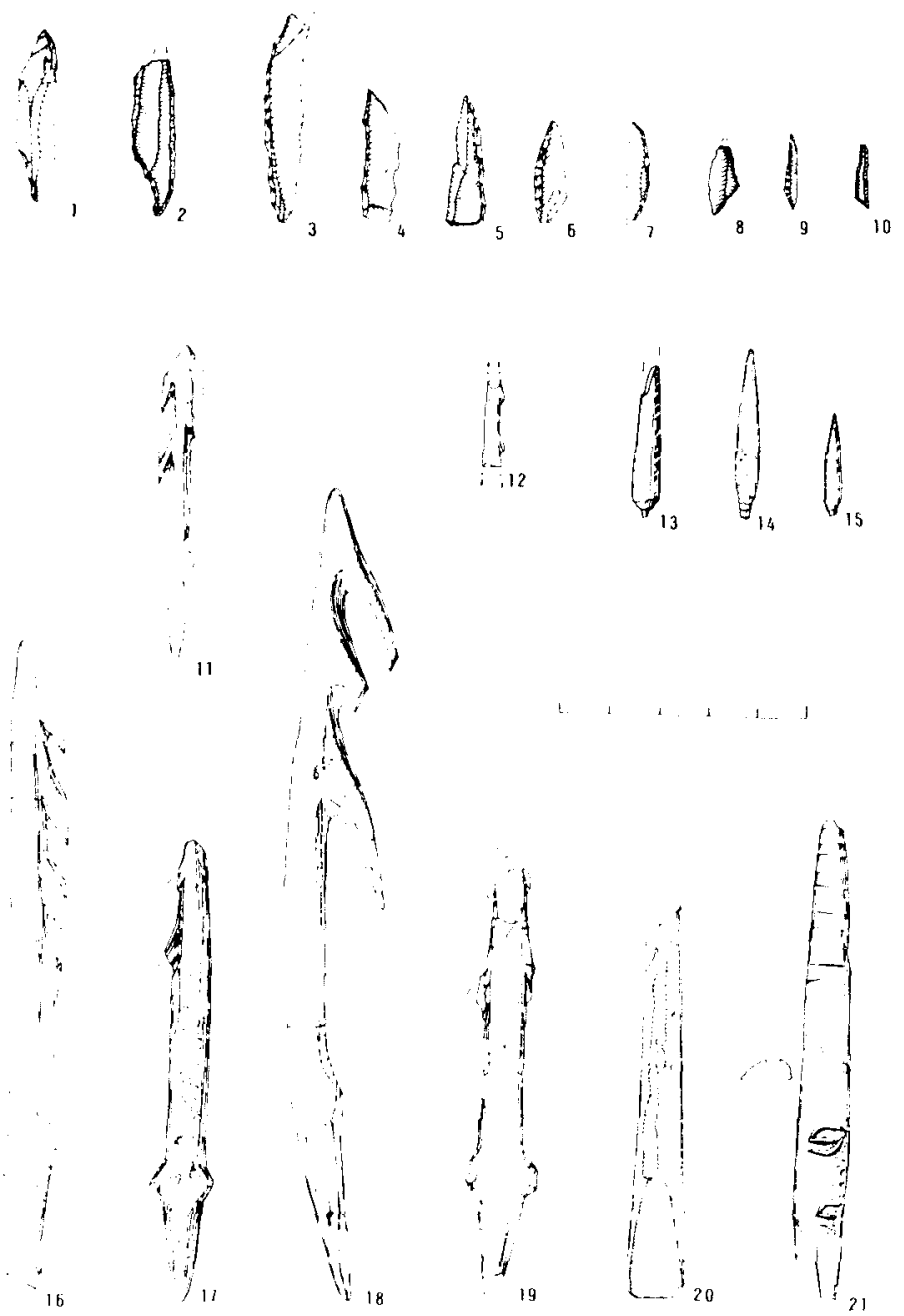

Fig. 7. Magdalénien supérieur et final, armatures en silex et en matière dure animale. 1 et 2 = pointes à cran, 3-6 = pointes à dos, $7=$ segment de cercle, $8-10=$ triangles, 11 et $19=$ têtes de harpons à barbelures bilatérales, $12=$ petite pointe barbelée, $16-18$ = têtes de harpons à barbelures unilatérales, 13 15 = petites pointes à base taillée et rompue, 20 = armature de sagaie à double biseau; 21 = élément d'armature de sagaie gravée, à section demi-ronde.

Cauna de Be/vis : 4 - 6, 9, 10, 12 - 15, 17, 19 - 21 (couches 1, 2, 3, fouilles $D$. Sacchi); Fontlaurier : 2 (récoltes E. Genson); grotte de l'Oeil : 8 et 11 (fouilles $D$. Sacchi et récoltes A. Abélanet); grotte du Trou Souffleur : 18 (récoltes A. Lachambre); La Teulera : 1 et 3 (récoltes J. Abélanet); Petite grotte de Bize : 16 (fouilles Th. et Ph. Héléna). 
1986) dominent le lot des armatures de trait. Par leurs faibles dimensions elles évoquent davantage des pointes de flèches que des extrêmités vulnérantes de lances et posent du même coup la question de l'acquisition de l'arc.

Au nombre des objets délaissés ou désormais fabriqués en des matériaux n'ayant pas résistés à l'épreuve du temps il faut mentionner le propulseur. Les spatules, dépourvues de décor, se raréfient. La technique du contour découpé ne s'applique plus qu'à la production de quelques pendeloques pisciformes (fig. $8, n .{ }^{\circ} 1$ ); ainsi disparaissent ces fameuses têtes d'ongulés antérieurement en faveur. Toujours dans le domaine de la parure, les perles de jay cylindriques (fig. $8, \mathrm{n}^{\circ}{ }^{2}$ ) se substituent aux spécimens globuleux antérièurs.

Les chasseurs du Magdalénien récent se montrèrent moins prolixes que leurs aînés en matière de décoration mobilière. Ils éprouverent toutefois la même nécessité de revêtir certains objets de signes ou de figures animales. L'art animalier cède fréquemment, dans la phase ultime de ce faciès culturel, au traitement schématique au point d'interdire, dans bien des cas, la détermination spécifique des sujets représentés (fig. 8, n. ${ }^{\circ}$ 3-6). Les cubitus de rapaces, transformés en tubes ou étuis, constituent alors, comme dans toute l'aire pyrénéenne, un support relativement commun. L'un des exemplaires fragmentés de Belvis montre un motif ovalaire qu'on peut interpréter comme une représentation de poisson passant à la figure strictement géométrique (fig. $8, n .^{\circ} 4$ ). Les hachures croisées figureraient ainsi les écailles dorsales selon une convention déjà adoptée pour deux des saumons réalistes du bâton de Lortet (Bandi et Maringer, 1952, fig. 63).

Quant à l'art rupestre il trouve sans doute sa meilleure sinon son unique expression dans le bestiaire gravé du rocher de Fornols-Haut (Sacchi et al., 1988) (fig. 8, n. ${ }^{\circ}$ 7-8). Par la manière, les dimensions, le recours à certains effets d'animation les figurations de Fornols rapellent précisément celles du Colombier II. Ces dernières, également éclairées par la lumière du jour, béneficient d'un contexte archéologique ne laissant aucun doute sur leur appartenance culturelle (Combier et al., 1984).

Toujours dominée par le renne dans les sites de basse vallée et de faible altitude (Tournal, La Crouzade) les faunes chassées font une large place au cheval. II s'y ajoute le bouquetin, dont les effectifs augmentent notablement en secteur escarpé ou montagneux (l'Oeil, Belvis), le chamois, des bovinés, le cerf, le lièvre parfois très abondant, etc...

Les poissons, exclusivement des salmonidés, s'affirment en territoire pyrénéen et justifie certainement la présence des plus petits modèles de 

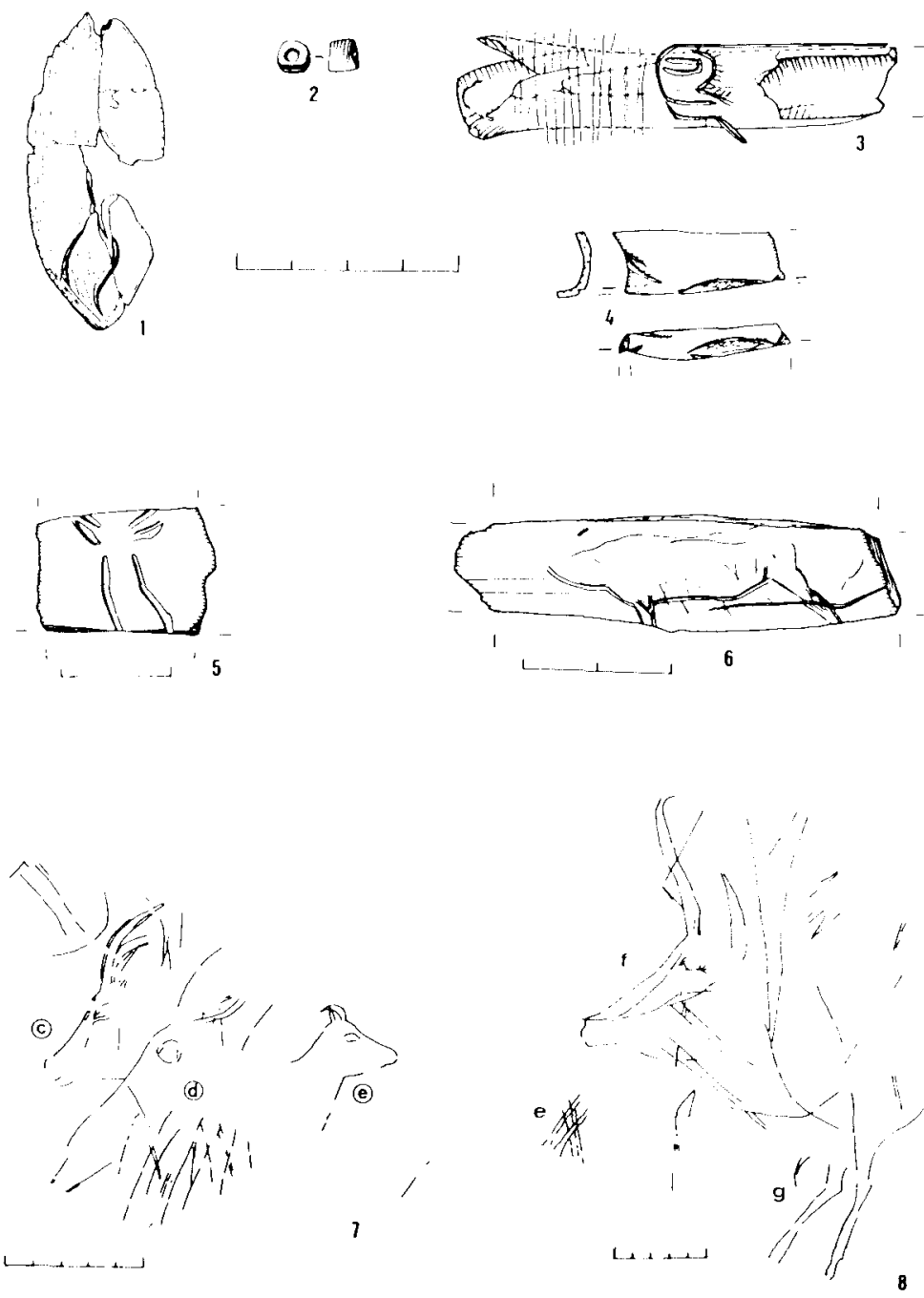

Fig. 8. Magdalénien supérieur et final, art mobilier et rupestre. $1=$ pendeloque ichtyoïde en os; 2 = perle cylindrique en jayet; 3 = tête d'ongulé affrontée à un réseau de traits gravés sur cubitus de Vautour fauve; 4 = motif pisciforme gravé sur cubitus de Vautour; 5 = tête et encolure de bouquetin (?) vues de face et gravées sur os poli; 6 = silhouette d'ongulé gravé sur fragment de diaphyse; $7=$ tête de bouquetin (c), tête d'ongulé indéterminé (d), tête de bouquelin inmature ou de jeune isard (e), gravées sur rocher; 8 = tête (f), arrière-train d'isard mâle (g) et signe claviforme (e) gravés sur rocher.

Cauna de Belvis: 1 - 6 (couches 3 et 4, fouilles D. Sacchi); Rocher de FornolsHaut : 7 et 8 (relevés $D$. Sacchi). (Dessins de l'auteur). 
harpon (fig. $7, n .{ }^{\circ} 11$ ) et des micro-pointes barbelées (fig. $7, n .{ }^{\circ} 12$ ), absentes des lieux dépourvus d'ichtyofaune.

Au regard des informations sur le paléoenvironnement, les sites les mieux documentés trahissent un froid sec qui n'atteint pas toutefois à l'intensité notée pendant le développement du Magdalénien moyen (Vilette, 1984). Mais au cours de cette phase corrélative au Dryas ancien supérieur de la zonation pollinique classique on note, à Belvis, l'existence d'un refuge d'arbres mésophiles et thermophiles (Jalut et al., 1975), confirmée par l'analyse des restes aviaires (Vilette, op. cit.).

\section{EN GUISE DE CONCLUSION}

De ces remarques générales il ressort en premier lieu que le bassin de l'Aude et le Roussillon connurent entre 15.000 et 12.000 B.P. une occupation humaine sans doute continue et d'une densité croissante. II apparaît en second lieu qu'après une coexistence vraisemblable des derniers Solutréens catalans et des premiers Magdaléniens/Badegouliens languedociens s'instaure, pour une durée de trois millénaires et sans partage, l'ère magdalénienne. En troisième lieu nous constatons l'existence, au sein de cette culture, de trois facies distincts et successifs. Le dernier d'entre-eux s'articulant en deux séquences continues.

Enfin il ne fait aucun doute que la contrée intéressée appartient en totalité, pour le moins à partir de 15.000 B.P. à la grande province culturelle pyrénéenne. 


\section{BIBLIOGRAPHIE}

BAHN, P., 1982: «Inter-site and inter-regional links during the upper palaeolithic: the pyrenean evidence", Oxford journal of Archaeology 1 (3), p. 247-268, 3 cartes, 6 fig.

BANDi, H. G. et MARINGer, J., 1952: L'Art préhistorique, Bâle et Paris 168 p., 216 fig.

BAZILE, F., 1977: "Le Magdalénien ancien de Camparnaud à Vers-Pontdu-Gard", Bulletin de la Société d'Etudes des Sciences Naturelles de Nimes, 55, p. 47-64, 7 fig.

Brochier, J. L., 1978: Les modifications de l'environnement de Würmien récent au Postglaciaire en Languedoc, CNRS, Marseille, 203 p., 101 fig., 2 tabl.

CLOTTES, J. (à paraître): “Le Magdalénien des Pyrénées", Actes du colloque "Le Magdalénien", XIlème Congrès de l'UISPP, Mayence 1987.

Combier, J., Porte, J. L., Ayroles, P. et Gely, B., 1984: “Abri du Colombier" in L'Art des Cavernes, atlas des grottes ornées paléolithiques françaises, Ministère de la Culture, 1984, p. 621-625, 3 fig.

Dupuis-Perez, I., 1986: "Les chiroptères de Gazel et Canecaude», extrait de Les chiroptères du Quaternaire en France, Mémoire de Maîtrise en Préhistoire, Université de Paris I, p. 192-247, 20 pl.

Gordon, B., 1988: Men and reindeer Herds in French Magdalenian Prehistory, BAR international Series 390, 333 p., 31 fig., 12 pl. photo.

Grazıosı, P., 1960: Palaeolithic art, Faber and Faber, Londres, 278 p., 41 fig. 2 dépl., 306 pl. h.t.

Jalut, G., Andrieu, V., Delibrias, G., et Pages, P., (sous presse): «Paleo environment of the valley of Ossau (French western Pyrenees) during the last 27000 years", Pollen et spores, 1988.

Jalut, J., SACCHI, D. et Vernet, J. L., 1975: "Mise en évidence d'un refuge tardiglaciaire à moyenne altitude sur le versant nord-oriental 
des Pyrénées (Belvis alt. $960 \mathrm{~m}$, Aude)». Compte rendus de l'Académie des Sciences de Paris, t. 280, série D., p. 1781-1784, 1 carte. SACCHI, D., 1967: "Le Paléolithique supérieur dans la Montagne Noire, états des recherches et perspectives", Atacina 1, p. 3-7.

- 1968: “Données nouvelles sur le Paléolithique supérieur du département de l'Aude», Atacina 2, 32 p., 12 fig.

- 1969: "Observations sur la stratigraphie de la Petite grotte de Bize», Atacina 4, p. 3-25, 6 fig.

- 1979: "Canecaude I (Villardonnel, Aude), grotte Gazel (Sallèles-Cabardès, Aude), Cauna de Belvis (Aude)" in Le Pléistocène supérieur en France, Illème Colloque Franco-Soviétique «Dynamique des Interactions entre le milieu naturel et les sociétés préhistoriques", Laboratoire de Paléontologie Humaine et de Préhistoire, Marseille, p. 184214, fig. 51-61.

- 1986: Le Paléolithique supérieur du Languedoc occidental et du Roussillon, Gallia-Préhistoire, XXlème suppl., Paris, CNRS, 284 p., 204 fig., XVI pl. h. t.

- (à paraître a): «18 000 - 11000 B.P. dans les Pyrénées septentrionales", in Le temps de la Préhistoire, Société préhistorique française, 1989.

- (à paraître b): «Bases objectives de la chronologie de l'art mobilier paléolithique dans les Pyrénées septentrionales", 1er Colloque International d'Art mobilier paléolithique, Foix Le Mas-d'Azil, 16 - 21 Novembre 1987.

Sacchi, D., avec la collaboration de Abelanet, J., Brule, J. J., Massiac, Y., Rubiella, C. et VILETTE, P., 1988: «Les gravures rupestres de Fornols-Haut, Pyrénées-orientales", L'Anthropologie, t. 92, n. ${ }^{\circ} 1, \mathrm{p}$. $87-100,19$ fig.

SONNEVILLE-Bordes, D. de, 1966: "L'évolution du Paléolithique supérieur en Europe occidentale et sa signification", Bulletin de la Société préhistorique française, LXIII, fasc. 1, p. 3 - 34 .

Tovoso, A., 1987: "Le remplissage de la grotte Tournal à Bize-Minervois (Aude)", Cypsela VI, p. 23 - 25, 35 fig.

VILETTE, P., 1984: “Avifaunes du Pléistocène final et de l'Holocène dans le Sud de la France et en Catalogne», Atacina 11 (1983), 190 p., 62 tabl., 15 cartes, 40 fig., 3 pl. h. t. 
